\title{
Pain Intervention for Cancer and Non-cancer Pain: A Retrospective Analysis of Tertiary Care Hospital Experience
}

Umair Ahmad ${ }^{1,2}$, Syed A. Abbas ${ }^{3,4}$, Syeda M. Hamadani ${ }^{4}$, Syed M. Abbas ${ }^{5}$, Samia Usman ${ }^{6}$, Zeeshan Hafeez ${ }^{7}$, Ateeq Ur Rehman Ghafoor ${ }^{8}$

1. Anaesthesia, Shaukat Khanum Memorial Cancer Hospital and Research Center, Lahore, PAK 2. Internal Medicine, Ittefaq Trust Hospital, Lahore, PAK 3. Internal Medicine, Montefiore Medical Center/Albert Einstein College of Medicine, Bronx, USA 4. Internal Medicine, Fatima Memorial Hospital College of Medicine and Dentistry, Lahore, PAK 5. Internal Medicine, Montefiore Medical Center, Wakefield Campus, Bronx, USA 6. Internal Medicine, King Edward Medical College, Lahore, PAK 7. Internal Medicine, Westchester Medical Center, Valhalla, USA 8. Anesthesia and Pain Management, Shaukat Khanum Memorial Cancer Hospital and Research Centre, Lahore, PAK

Corresponding author: Umair Ahmad, umairahmed.316@gmail.com

\section{Abstract}

\section{Background}

With the recent advancement in medicine there has been a great emphasis on the management of chronic pain which remains as one of the major contributing factors for functional limitation in patients as well as a financial burden on healthcare. Newer treatment modalities are aimed at terminating the vicious pain cycles and in this regard peripheral nerve blocks have proven to be very effective.

\section{Objectives}

The aim of this study is to evaluate the effectiveness of interventions for both cancer and non-cancer patients by objective assessment of the patients before and after the procedure.

\section{Materials and methods}

The study included 252 patients who underwent nerve block procedures in Shaukat Khanum Memorial Cancer Hospital from December 2016 to December 2018. The patients were evaluated using numerical rating scale (NRS) for pain, reduction in analgesic doses and patient satisfaction after one and four weeks post procedure. The data was analyzed using mean values and calculating percentages.

\section{Results}

Received 04/08/2020 Review began 04/10/2020 Review ended 04/11/2020 Published 04/18/2020

() Copyright 2020

Ahmad et al. This is an open access article distributed under the terms of the Creative Commons Attribution License CC-BY 4.0., which permits unrestricted use, distribution, and reproduction in any medium, provided the original author and source are credited.
In cancer group, 168 patients were included; mean age $50.49 \pm 15.39$ with $46.43 \%$ females and $53.57 \%$ males, the average pain score was $2.62 \pm 1.87$ post procedure compared with $6.30 \pm 1.87$ post procedure. $48.21 \%$ of the patients reported a reduction in analgesia while $51.79 \%$ of the patients kept on using the same analgesics doses. $74.40 \%$ of the patients were satisfied and $25.60 \%$ patients remained unsatisfied after one week whereas $66.07 \%$ were satisfied, $23.81 \%$ were not satisfied and $10.12 \%$ loss to follow up after four weeks. In non-cancer group 84 patients were included; mean age $56.49 \pm 15.79$ with $41.67 \%$ females and $58.33 \%$ males, the average pain score before intervention was $5.99 \pm 1.21$ and after intervention it was $2.43 \pm 1.62$. In $73.81 \%$ non-cancer patients the analgesics doses were reduced and $70.24 \%$ patients were satisfied while $29.76 \%$ were unsatisfied after one week. After four weeks $55.95 \%$ were satisfied, $22.62 \%$ were not satisfied and $21.43 \%$ loss to follow up.

\section{Conclusion}

The study showed decrease in pain scores in both group of patients and the importance of nerve blocks as an effective method for chronic pain management. The reduction in the use of other analgesics was also commendable in both the groups.

Categories: Anesthesiology, Pain Management, Oncology

Keywords: chronic pain, pain management, cancer and non cancer pain

\section{Introduction}

With the recent advancement in medicine there has been a great emphasis on the management of chronic pain which remains as one of the major contributing factors for functional limitation in patients and poor quality of life as well as a financial burden on healthcare. The prevalence of chronic pain is estimated to be $37.3 \%$ in developed and $41.1 \%$ in developing countries [1]. 


\section{Cureus}

In 2012, there were more than 14 million diagnosed cancer patients and it is estimated that it will rise to more than 20 million by 2025 [2]. With better treatment options more patients are surviving with cancer and one of the fearsome aspects of this disease is chronic pain which even at present is very challenging to manage. Although the use of opioids has greatly helped in reducing the pain associated with the disease but still the prevalence of chronic pain remains high.

Similarly non-cancer chronic pain is very common and its prevalence was estimated to be $19 \%$ in Europe [3]. The American Pain Society survey also has estimated that $9 \%$ of the adult population suffers from moderate to severe, non-cancer related pain [4]. Epidemiological data in the elderly population estimates that up to $50 \%$ of them suffer from chronic pain [5].

In the past few decades, the mainstay management for chronic pain was opioids and topical therapies. World Health Organization (WHO) also recommends use of opioids as part of the analgesic step-ladder approach but prolonged use of opioid medication is associated with serious side effects and patient compliance is an issue. Recently, researchers are focused towards discovering the changes that happen in brain and nervous system because of chronic pain to better understand the physical basis and to establish better treatment options. Newer treatment modalities are aimed at terminating the vicious pain cycles and in this regard nerve blocks have proven to be very effective.

Previously an abstract was presented which showed the efficacy of interventional pain procedures for cancer and non-cancer pain (Poster presentation: Ahmad U. Pain Interventions for Cancer and Non-Cancer Pain: A Retrospective Analysis of Shaukat Khanum Memorial Cancer Hospital and Research Centre Experience. 17th Shaukat Khanum Cancer Symposium; Nov 2-4, 2018). The primary objective of this study is to emphasize the role of interventional procedures along with the pharmacological therapy in pain management.

\section{Materials And Methods}

In this study, retrospective data of patients was analyzed who underwent interventional pain procedures at Shaukat Khanum Memorial Cancer Hospital and Research Centre from December 2016 to December 2018. The data was collected after getting Institutional Review Board (IRB) approval. The patients were evaluated using numerical rating scale (NRS) for pain (Figure 1), reduction in analgesic doses and patient satisfaction after one and four weeks post procedure. The patient satisfaction criteria were based on reduction in pain score according to NRS and the improvement in carrying out daily routine activities (Tables 1, 2). The data was analyzed using mean values and calculating percentages.

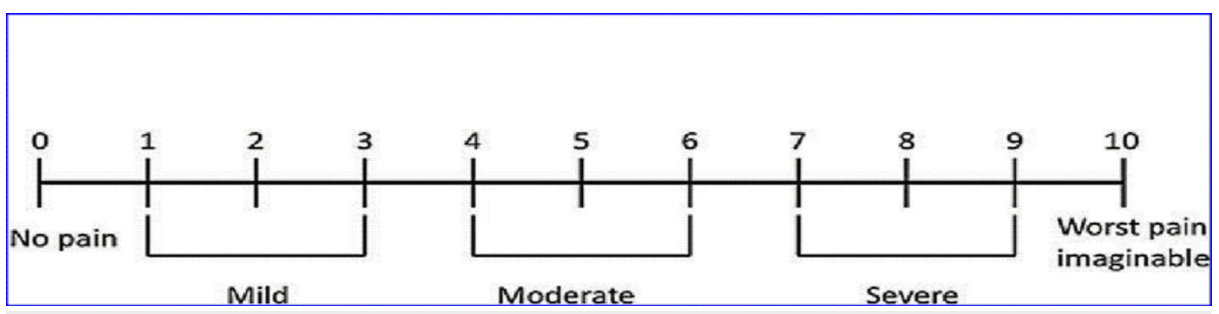

FIGURE 1: Numerical Rating Scale (NRS)

\begin{tabular}{|c|c|c|c|c|c|c|c|c|c|}
\hline \multicolumn{10}{|c|}{ Pre Procedure Pain Score } \\
\hline 1 & 2 & 3 & 4 & 5 & 6 & 7 & 8 & 9 & 10 \\
\hline \multicolumn{10}{|c|}{ Post Procedure Pain Score } \\
\hline 1 & 2 & 3 & 4 & 5 & 6 & 7 & 8 & 9 & 10 \\
\hline & 70 & & & & & & & & \\
\hline
\end{tabular}




\section{Cureus}

\section{Would you undergo repeat procedure if required?}

Do you feel more comfortable in carrying out daily activities post procedure?

Do you have to wake up at night due to pain post procedure?

Do you feel more refreshed in the morning post procedure?

\section{TABLE 2: Satisfaction Score Table}

\section{Results}

The study included 252 patients which were further divided into two groups: cancer and non-cancer patients. In cancer group 168 patients were included; their mean age was $50.49 \pm 15.39$ with $46.43 \%$ females and $53.57 \%$ males, the average pain score was $2.62 \pm 1.87$ after the procedure compared with $6.30 \pm 1.87$ before the intervention. $48.21 \%$ of the patients reported a reduction in analgesic doses while $51.79 \%$ of the patients kept on using the same analgesics doses. $74.40 \%$ of the patients were satisfied and $25.60 \%$ patients remained unsatisfied after one week whereas $66.07 \%$ were satisfied, $23.81 \%$ were not satisfied and $10.12 \%$ loss to follow up after four weeks (Tables 3-6).

Total number of patients

Pain score before procedure

Pain score after procedure

Reduction in analgesic medication dose reported by patients

Overall Satisfaction after 1 week

Overall Satisfaction after 4 weeks
168

\subsection{0 (NRS)}

2.62 (NRS)

81

$74.40 \%$

$66.07 \%$

\section{TABLE 3: Pain Interventional Procedures in Cancer Patients}

NRS: Numerical pain score

\section{Total number of patients}

Pain score before procedure

Pain score after procedure

Reduction in analgesic medication dose reported by patients

Overall Satisfaction after 1 week

Overall Satisfaction after 4 weeks
40

7.1 (NRS)

2.8 (NRS)

25

$75 \%$

$55 \%$

\section{TABLE 4: Coeliac Plexus Block in Cancer Patients}

NRS: Numerical pain score 


\section{Cureus}

Total number of patients

Pain score before procedure

Pain score after procedure

Reduction in analgesic medication dose reported by patients

Overall Satisfaction after 1 week

TABLE 5: Epidural Rhizolysis in Cancer Patients

NRS: Numerical pain score

Total number of patients

30

Pain score before procedure

5.5 (NRS)

Pain score after procedure

2.7 (NRS)

Reduction in analgesic medication dose reported by patients

Overall Satisfaction after 1 week

$60 \%$

Overall Satisfaction after 4 weeks
$70 \%$

5

\section{TABLE 6: Intrathecal Neurolysis in Cancer Patients}

NRS: Numerical pain score

In non-cancer group 84 patients were included with mean age of $56.49 \pm 15.79$ with $41.67 \%$ females and $58.33 \%$ males, the average pain score before intervention was $5.99 \pm 1.21$ and after intervention it was $2.43 \pm$ 1.62. In $73.81 \%$ non-cancer patients the analgesics doses were reduced and $70.24 \%$ patients were satisfied while $29.76 \%$ were unsatisfied after one week. After four weeks $55.95 \%$ were satisfied, $22.62 \%$ were not satisfied and $21.43 \%$ loss to follow up (Tables 7-10).

Total number of patients

Pain score before procedure

Pain score after procedure

Reduction in analgesic medication dose reported by patients

Overall Satisfaction after 1 week

Overall Satisfaction after 4 weeks
84

5.99 (NRS)

2.43 (NRS)

62

$70.24 \%$

$55.95 \%$

\section{TABLE 7: Pain Interventional Procedures in Non-Cancer Patients}

NRS: Numerical pain score 


\section{Cureus}

Total number of patients

Pain score before procedure

Pain score after procedure

Reduction in analgesic medication dose reported by patients

Overall Satisfaction after 1 week

TABLE 8: Intra-articular Injection in Non-Cancer Patients

NRS: Numerical pain score

Total number of patients

32

Pain score before procedure

6.2 (NRS)

Pain score after procedure

2.4 (NRS)

Reduction in analgesic medication dose reported by patients

26

Overall Satisfaction after 1 week

$81.25 \%$

Overall Satisfaction after 4 weeks

$62.5 \%$

\section{TABLE 9: Epidural Rhizolysis in Non-Cancer Patients}

NRS: Numerical pain score

\begin{tabular}{|l|c|}
\hline Total number of patients & 6 \\
\hline Pain score before procedure & 6.8 (NRS) \\
\hline Pain score after procedure & 2.4 (NRS) \\
\hline Reduction in analgesic medication dose reported by patients & 3 \\
\hline Overall Satisfaction after 1 week & $66.66 \%$ \\
\hline Overall Satisfaction after 4 weeks & $50 \%$
\end{tabular}

\section{TABLE 10: Ganglion Impar Block in Non-Cancer Patients}

NRS: Numerical pain score

\section{Discussion}

Chronic pain remains as one of the major factors that has a negative impact on patient's physical and psychological health. Chronic pain not only adversely affects the patient but also their families. The WHO analgesic ladder provides the basic guidelines to address chronic pain depending on the disease severity. Most of the patients are on opioids for pain control with variable tolerance to medication. Although opioids provide good pain relief for these patients, but opioids do have side effects and if not managed properly they can be a reason for non-compliance and poor quality of life. Also, with the prolonged use there are tolerance and dependency issues. Nerve blocks have been used for pain management for over a century now. In 1884, Koller first reported the use of nerve blocks [6]. They can be used either with local anesthetics or neurolytic agents. Nerve blocks act by inhibiting the impulse transmission from the peripheral nerve ending resulting in termination of the pain signal perceived by the cortex. 
In this study, we have evaluated the efficacy of nerve blocks in chronic pain management, reduction in analgesic doses and patient satisfaction post procedure. The study included 252 patients out of which 168 are cancer patients and 84 non-cancer patients. The patients underwent different pain interventional procedures and numerical pain scores were used to assess the efficacy of the treatment.

In cancer patients, most of the procedures were carried out with palliative intent to control the pain and improve the quality of life in terminal patients. Few of the patients have to undergo repeat procedures for adequate pain control. The prevalence of pain in cancer patients with advanced stage is around $62 \%-86 \%$ which emphasize that adequate pain control is not achieved in majority of the patients [7-10].

Most common procedure carried out in our centre was coeliac plexus block (40), epidural rhizolysis (40) and intrathecal neurolysis (30) in cancer patients. Coeliac plexus block is carried out in patients with intractable pain in pancreatic and upper abdominal organ carcinomas. Recently a study was published which emphasized on the efficacy of these blocks for upper abdominal cancer pain [11]. Most of the patients reported adequate pain control with the intervention as documented in previous studies $[9,12,13]$. The requirement of other analgesic medications was not significantly decreased in these patients. Few of the patients had to undergo a repeat procedure because of the underlying disease progression for adequate pain control [14].

In epidural rhizolysis the nerves carrying sensation to the spinal cord are desensitized by using a combination of local anaesthetic and steroid so that pain sensation is reduced. Epidural injections have been carried out since 1900s for relieving back pain. The efficacy of this procedure in reducing pain is well established in patients with refractory cancer pain $[15,16]$. At our centre a total of 59 epidural rhizolysis procedures were carried out both for cancer and non-cancer pain at different spinal levels. There have been debates regarding efficacy and complications related to epidural injections but in our centre majority of the patients reported good pain relief post procedure.

The procedure for intrathecal neurolysis was first described by Dogliotti in 1931 and has been used since then for intractable cancer pain. Careful selection of the patients is needed as some serious complications are associated with the procedure. The chemical neurolysis had been carried out with different agents including alcohol and phenol-glycerol combination with similar pain relief results in patients [17-19]. Likewise with other pain interventional procedures there was a significant reduction in pain scores as analyzed by the numerical pain score (NRS) and around $74.40 \%$ patients were satisfied in terms of pain control after one week post procedure.

For non-cancer pain intra-articular injection (32), epidural rhizolysis (32) and ganglion impar blocks (6) were the most common procedures. Musculoskeletal pain is the most common type of chronic pain seen in the adult population. In US adults the prevalence of doctor-diagnosed arthritis was $21 \%$ (46.4 million persons) [20]. The pain due to joint disease is among the top 10 causes of disability worldwide [21]. Intra-articular corticosteroid and hyaluronic acid injections are used if the pharmacological therapy is not effective.

Ganglion impar block (GIB) was first described in 1990 and was primarily used for pain control in cancer patients. Since then the procedure is commonly performed for pain in the terminal segment of the spine near the coccyx and perineal area also referred to as Coccydynia. The blockade of nociceptive and sympathetic fibers is achieved by this block which helps in pain relief [22]. Various methods and techniques have been described for this procedure. A number of studies have been conducted to show the efficacy and safety of the block in relieving perineal pain [22-24]. In non-cancer group $73.81 \%$ reported reduction in analgesic doses as compared to cancer group which showed only $48.21 \%$. Although the patient satisfaction after one week post procedure was similar in both the groups.

\section{Conclusions}

Pain interventional procedures showed much better pain control and patient satisfaction in both cancer and non-cancer group as compared to conventional pharmacological therapy. Interventional procedures can be used as an adjuvant to pharmacological therapy and also will help in reducing opioid dose and their side effects.

\section{Additional Information}

\section{Disclosures}

Human subjects: Consent was obtained by all participants in this study. Institutional Review Board Shaukat Khanum Memorial Cancer Hospital and Research Centre issued approval EXMPT-01-10-18-04-A1. Animal subjects: All authors have confirmed that this study did not involve animal subjects or tissue. Conflicts of interest: In compliance with the ICMJE uniform disclosure form, all authors declare the following: Payment/services info: All authors have declared that no financial support was received from any organization for the submitted work. Financial relationships: All authors have declared that they have no financial relationships at present or within the previous three years with any organizations that might have an interest in the submitted work. Other relationships: All authors have declared that there are no other 


\section{References}

1. Tsang A, Von Korff M, Lee S, et al.: Common chronic pain conditions in developed and developing countries: gender and age differences and comorbidity with depression-anxiety disorders. J Pain. 2008, 9:883-891. 10.1016/j.jpain.2008.05.005

2. Ferlay J, Soerjomataram I, Dikshit R, et al.: Cancer incidence and mortality worldwide: sources, methods and major patterns in GLOBOCAN 2012. Int J Cancer. 2015, 136:359-386. 10.1002/ijc.29210

3. Reid KJ, Harker J, Bala MM, Truyers C, Kellen E, Bekkering GE, Kleijnen J: Epidemiology of chronic noncancer pain in Europe: narrative review of prevalence, pain treatments and pain impact. Curr Med Res Opin. 2011, 27:449-462. 10.1185/03007995.2010.545813

4. Rovine T, Ferrero CL: Chronic Pain in America: Roadblocks to Relief . American Pain Society, Glenview; 1999.

5. Sawyer P, Bodner EV, Ritchie CS, Allman RM: Pain and pain medication use in community-dwelling older adults. Am J Geriatr Pharmacother. 2006, 4:316-324. 10.1016/j.amjopharm.2006.12.005

6. Koller C: On the use of cocaine for producing anaesthesia on the eye . Lancet. 1884, 124:990-992. 10.1016/S0140-6736(02)28859-5

7. Di Maio M, Gridelli C, Gallo C, et al.: Prevalence and management of pain in Italian patients with advanced non-small-cell lung cancer. Br J Cancer. 2004, 90:2288-2296. 10.1038/sj.bjc.6601810

8. Wilson KG, Graham ID, Viola RA, Chater S, de Faye BJ, Weaver LA, Lachance JA: Structured interview assessment of symptoms and concerns in palliative care. Can J Psychiatry. 2004, 49:350-358. $10.1177 / 070674370404900603$

9. Hwang SS, Chang VT, Cogswell J, et al.: Study of unmet needs in symptomatic veterans with advanced cancer: incidence, independent predictors and unmet needs outcome model. J Pain Symptom Manage. 2004, 28:421-432. 10.1016/j.jpainsymman.2004.02.023

10. Stromgren AS, Groenvold M, Petersen MA, et al.: Pain characteristics and treatment outcome for advanced cancer patients during the first week of specialized palliative care. J Pain Symptom Manage. 2004, 27:104113. 10.1016/j.jpainsymman.2003.06.011

11. Nagels W, Pease N, Bekkering G, Cools F, Dobbels P: Celiac plexus neurolysis for abdominal cancer pain: a systematic review. Pain Med. 2013, 14:1140-1163. 10.1111/pme.12176

12. Kawamata M, Ishitani K, Ishikawa K, Sasaki H, Ota K, Omote K, Namiki A: Comparison between celiac plexus block and morphine treatment on quality of life in patients with pancreatic cancer pain. Pain. 1996, 64:597602. 10.1016/0304-3959(95)00189-1

13. Polati E, Finco G, Gottin L, Bassi C, Pederzoli P, Ischia S: Prospective randomized double-blind trial of neurolytic coeliac plexus block in patients with pancreatic cancer. Br J Surg. 1998, 85:199-201. 10.1046/j.1365-2168.1998.00563.x

14. McGreevy K, Hurley RW, Erdek MA, Aner MM, Li S, Cohen SP: The effectiveness of repeat celiac plexus neurolysis for pancreatic cancer: a pilot study. Pain Pract. 2013, 13:89-95. 10.1111/j.1533-2500.2012.00557.x

15. Burton AW, Rajagopal A, Shah HN, Mendoza T, Cleeland C, Hassenbusch SI III, Arens JF: Epidural and intrathecal analgesia is effective in treating refractory cancer pain. Pain Med. 2004, 5:239-247. 10.1111/j.1526-4637.2004.04037.x

16. Jeon YS, Lee JA, Choi JW, et al.: Efficacy of epidural analgesia in patients with cancer pain: a retrospective observational study. Yonsei Med J. 2012, 53:649-653. 10.3349/ymj.2012.53.3.649

17. Koyyalagunta D, Burton A: The role of chemical neurolysis in cancer pain . Curr Pain Headache Rep. 2010, 14:261-267. 10.1007/s11916-010-0123-9

18. Candido K, Philip C, Ghaly R, Knezevic N: Transforaminal 5\% phenol neurolysis for the treatment of intractable cancer pain. Anesth Analg. 2010, 110:216-219. 10.1213/ANE.0b013e3181c0ecd5

19. Watanabe A, Yamakage M: Intrathecal neurolytic block in a patient with refractory cancer pain . J Anesth. 2011, 25:603-605. 10.1007/s00540-011-1141-4

20. Helmick CG, Felson DT, Lawrence RC, et al.: Estimates of the prevalence of arthritis and other rheumatic conditions in the United States: Part I. Arthritis Rheum. 2008, 58:15-25. 10.1002/art.23177

21. The National Collaborating Centre for Chronic Conditions: Osteoarthritis: National Clinical Guideline for Care and Management in Adults. Royal College of Physicians, London; 2008.

22. Toshniwal GR, Dureja GP, Prashanth SM: Transsacrococcygeal approach to ganglion impar block for management of chronic perineal pain: a prospective observational study. Pain Physician. 2007, 10:661-666.

23. Reig E, Abejón D, del Pozo C, Insausti J, Contreras R: Thermocoagulation of the ganglion impar or ganglion of Walther: description of a modified approach. Preliminary results in chronic, nononcological pain. Pain Pract. 2005, 5:103-110. 10.1111/j.1533-2500.2005.05206.x

24. Agarwal-Kozlowski K, Lorke DE, Habermann CR, Am Esch JS, Beck H: CT-guided blocks and neuroablation of the ganglion impar (Walther) in perineal pain: anatomy, technique, safety, and efficacy. Clin J Pain. 2009, 25:570-576. 10.1097/AJP.0b013e3181a5f5c7 